\title{
Optimal Stopping of a Killed Exponentially Growing Process
}

\author{
Fredrik Armerin*
}

\begin{abstract}
We consider a finite horizon optimal stopping problem. The value of the underlying process grows exponentially until a Poisson process jumps for the first time, at which the processes jumps to zero and stays there forever. As applications of this model we consider valuing real options and options written on the stock of a start-up company.
\end{abstract}

Keywords: Optimal stopping, Poisson processes, American options.

*Department of Real Estate and Construction Management, Royal Institute of Technology KTH, Sweden. Email: fredrik.armerin@abe.kth.se. 


\section{Introduction}

In the main part of this paper we consider an optimal stopping problem with a finite horizon where the underlying stochastic process grows exponentially with a constant rate until it hits zero and then stays there forever. To show the applicability of this type of models, we consider two applications from finance: The valuation of a real option when competition is present, and the valuing options written on a start-up company.

The rest of the paper is organized as follows. In Section 2 the basic model and results are presented. Section 3 contains the solution to the optimal stopping problem, and in Section 4 the model is applied to the problem of valuing options written on the stock of a start-up.

\section{The model}

We let $\left(\Omega, \mathcal{F},\left(\mathcal{F}_{t}\right), P\right)$ be a complete filtered probability space. The filtration is assumed to be right-continuous and $\mathcal{F}_{0}$ contains all null sets of $\mathcal{F}$. We recall that a stopping time (with respect to the filtration $\left(\mathcal{F}_{t}\right)$ ) is a random variable $\tau: \Omega \rightarrow[0, \infty]$ with the property that

$$
\{\tau \leq t\} \in \mathcal{F}_{t} \text { for every } t \in \mathbb{R}_{+} .
$$

For $0 \leq t \leq T$ we let

$$
\mathcal{S}_{t, T}=\{\text { Stopping times taking values in }[t, T]\} .
$$

The underlying stochastic process in our model satisfies the stochastic differential equation

$$
d X_{t}=\mu X_{t} d t-X_{t-} d N_{t},
$$

where $\mu>0$ and $N$ is a Poisson process with constant intensity $\lambda>0$. Hence, the process $\left(X_{t}\right)$ grows exponentially until the first jump of the Possion process, at which time it jumps to zero and stays at zero forever. For $0 \leq t \leq s$ the solution of (1) can be written

$$
X_{s}=X_{t} e^{\mu(s-t)} \mathbf{1}\left(N_{s}=0\right) .
$$

We let $P_{t, x}$ denote the probability measure defined by

$$
P_{t, x}(\cdot)=P\left(\cdot \mid X_{t}=x\right),
$$

and let $E_{t, x}$ denote the expected value with respect to the measure $P_{t, x}$. It holds that

$$
E_{t, x}\left[X_{s}\right]=x e^{(\mu-\lambda)(s-t)},
$$

and more generally we have the following lemma. 
Lemma 2.1 If $\left(X_{t}\right)$ satisfies Equation (1), then for any measurable $g: \mathbb{R} \rightarrow \mathbb{R}$ with $g(0)=0$ and $0 \leq t \leq s$ we have

$$
E_{t, x}\left[g\left(X_{s}\right)\right]=g\left(x e^{\mu(s-t)}\right) e^{-\lambda(s-t)} .
$$

Proof. Using

$$
X_{s}=X_{t} e^{\mu(s-t)} \mathbf{1}\left(N_{s}=0\right)
$$

we get

$$
g\left(X_{s}\right)=g\left(X_{t} e^{\mu(s-t)} \mathbf{1}\left(N_{s}=0\right)\right) .
$$

Given $X_{t}=x$ the random variable $g\left(X_{s}\right)$ is bounded by $\left|g\left(x e^{\mu s}\right)\right|$, so $g\left(X_{s}\right)$ is integrable with respect to $P_{t, x}$. Now, since $g(0)=0$,

$$
\begin{aligned}
E_{t, x}\left[g\left(X_{s}\right)\right] & =E_{t, x}\left[g\left(x e^{\mu(s-t)} \mathbf{1}\left(N_{s}=0\right)\right)\right] \\
& =E_{t, x}\left[g\left(x e^{\mu(s-t)}\right) \mathbf{1}\left(N_{s}=0\right)\right] \\
& =g\left(x e^{\mu(s-t)}\right) E\left[\mathbf{1}\left(N_{s-t}=0\right)\right] \\
& =g\left(x e^{\mu(s-t)}\right) e^{-\lambda(s-t)} .
\end{aligned}
$$

\section{The optimal stopping problem}

For a fixed time $T>0$ and a constant $K>0$ we want to find

$$
V(t, x)=\sup _{\tau \in \mathcal{S}_{t, T}} E_{t, x}\left[e^{-\rho(\tau-t)} \max \left(X_{\tau}-K, 0\right)\right],
$$

where $\left(X_{t}\right)$ satisfies Equation (1) and $\rho>0$. We also, for each $t \in[0, T]$, want to find an optimal stopping time, i.e. a stopping time $\tau_{t}^{\star}$ satisfying

$$
V(t, x)=E_{t, x}\left[e^{-\rho\left(\tau_{t}^{\star}-t\right)} \max \left(X_{\tau_{t}^{\star}}-K, 0\right)\right] .
$$

In a financial context, this is, in principle, the value of an American call option. Depending on valuation approach, the probability measure under which the expected value is taken and which discount rate to use differs; see Dixit \& Pindyck [2] for details.

The value of the optimal stopping problem when $T=\infty$ is well known. Due to time homogeneity the value at time $t \geq 0$ of this optimal stopping problem is given by $V_{\infty}\left(X_{t}\right)$, where

$$
V_{\infty}(x)=\sup _{\tau} E_{0, x}\left[e^{-\rho \tau} \max \left(X_{\tau}-K, 0\right)\right],
$$


and where the supremum is taken over the set of stopping times. One can show that when $\rho>0$ and $\rho+\lambda>\mu$, then

$$
V_{\infty}(x)= \begin{cases}\left(\frac{x}{X_{c}}\right)^{\frac{\rho+\lambda}{\mu}}\left(X_{c}-K\right) & \text { if } 0 \leq x<X_{c} \\ x-K & \text { if } x \geq X_{c}\end{cases}
$$

where

$$
X_{c}=K \frac{\rho+\lambda}{\rho+\lambda-\mu} .
$$

For a proof of this, see e.g. Section 5.B in Dixit \& Pindyck [2]. We let $V_{E}$ denote the function

$$
V_{E}(t, x)=E_{t, x}\left[e^{-\rho(T-t)} \max \left(X_{T}-K, 0\right)\right] .
$$

Using Lemma 2.1 above with $g(x)=\max (x-K, 0)$ we get

$$
\begin{aligned}
V_{E}(t, x) & =e^{-(\rho+\lambda)(T-t)} \max \left(x e^{\mu(T-t)}-K, 0\right) \\
& =e^{-(\rho+\lambda-\mu)(T-t)} \max \left(x-K e^{-\mu(T-t)}, 0\right) .
\end{aligned}
$$

We will now proceed formally, abstaining from mathematical rigor, to arrive at a candidate solution to the optimal stopping problem. We then prove (see Theorem 3.1) that this is indeed the correct solution. Starting with the stochastic process $V\left(t, X_{t}\right)$ and using Ito's formula for processes of finite variation on $e^{-\rho t} V\left(t, X_{t}\right)$, assuming that $V\left(t, X_{t}\right)$ is regular enough for this to work, yields

$$
\begin{aligned}
d\left(e^{-\rho t} V\left(t, X_{t}\right)\right)= & -\rho e^{-\rho t} V\left(t, X_{t}\right) d t \\
& +e^{-\rho t}\left(\frac{\partial V}{\partial t}\left(t, X_{t}\right) d t+\frac{\partial V}{\partial x}\left(t, X_{t}\right) d X_{t}^{c}+V\left(t, X_{t}\right)-V\left(t, X_{t-}\right)\right) \\
= & e^{-\rho t}\left(-\rho V\left(t, X_{t}\right)+\frac{\partial V}{\partial t}\left(t, X_{t}\right)+\mu X_{t} \frac{\partial V}{\partial x}\left(t, X_{t}\right)\right) d t \\
& -e^{-\rho t} V\left(t, X_{t-}\right) d N_{t} \\
= & e^{-\rho t}\left(-(\rho+\lambda) V\left(t, X_{t}\right)+\frac{\partial V}{\partial t}\left(t, X_{t}\right)+\mu X_{t} \frac{\partial V}{\partial x}\left(t, X_{t}\right)\right) d t \\
& -e^{-\rho t} V\left(t, X_{t-}\right) d M_{t},
\end{aligned}
$$

where $M_{t}:=N_{t}-\lambda t$. We now integrate this from $t$ to $T$ and take the expected value with respect to $P_{t, x}$. If the process

$$
\int_{0}^{t} e^{-\rho u} V\left(u, X_{u-}\right) d M_{u}
$$

is a true martingale and using that $V(T, x)=\max (x-K, 0)$ we get

$$
\begin{aligned}
V(t, x)= & e^{-\rho(T-t)} E_{t, x}\left[\max \left(X_{T}-K, 0\right)\right] \\
& +\int_{t}^{T} e^{-\rho(s-t)} E_{t, x}\left[\left(-(\rho+\lambda) V\left(s, X_{s}\right)+\frac{\partial V}{\partial t}\left(s, X_{s}\right)+\mu X_{s} \frac{\partial V}{\partial x}\left(s, X_{s}\right)\right) d s\right] .
\end{aligned}
$$


Assuming that there exists a function $b(t)$, the optimal exercise boundary, such that

$$
V(t, x)=x-K \text { when } x \geq b(t)
$$

and

$$
\frac{\partial V}{\partial t}(t, x)+\mu x \frac{\partial V}{\partial x}(t, x)-(\rho+\lambda) V(t, x)=0 \text { for }(t, x) \text { such that } x<b(t)
$$

(see Peskir \& Shiryaev [4] for a motivation why these relations could be expected to hold) gives

$$
\begin{aligned}
V(t, x)= & e^{-\rho(T-t)} E_{t, x}\left[\max \left(X_{T}-K, 0\right)\right] \\
& +\int_{t}^{T} e^{-\rho(s-t)} E_{t, x}\left[\left((\rho+\lambda-\mu) X_{s}-(\rho+\lambda) K\right) \mathbf{1}\left(X_{s}>b(s)\right)\right] d s
\end{aligned}
$$

Under $P_{t, x}$ we have

$$
\mathbf{1}\left(X_{s}>b(s)\right)=1 \text { if and only if } x>b(s) e^{-\mu(s-t)} \text { and } N_{s}=0 .
$$

Hence, the integral on the right-hand side of Equation (2) is equal to

$$
\int_{t}^{T} e^{-(\rho+\lambda)(s-t)}\left((\rho+\lambda-\mu) x e^{\mu(s-t)}-(\rho+\lambda) K\right) \mathbf{1}\left(x e^{\mu(s-t)}>b(s)\right) d s .
$$

Now, if for $t \in[0, T]$ we have $X_{t}=b(t)$, then it is optimal to stop immediately. This means that $V(t, b(t))=b(t)-K$ for any $t \in[0, T]$. Choosing $x=b(t)$ in Equation (2) leads to the equation

$$
\begin{aligned}
b(t)-K= & e^{-(\rho+\lambda)(T-t)}\left(b(t) e^{\mu(T-t)}-K\right) \\
& +\int_{t}^{T} e^{-(\rho+\lambda)(s-t)}\left((\rho+\lambda-\mu) b(t) e^{\mu(s-t)}-(\rho+\lambda) K\right) \mathbf{1}\left(b(t) e^{\mu(s-t)}>b(s)\right) d s
\end{aligned}
$$

for $b(t)$. We now make the 'ansatz' $b(t)=A>0$. Inserting this results in the equation yields

$$
\begin{aligned}
A-K= & e^{-(\rho+\lambda)(T-t)}\left(A e^{\mu(T-t)}-K\right) \\
& +\int_{t}^{T} e^{-(\rho+\lambda)(s-t)}\left((\rho+\lambda-\mu) A e^{\mu(s-t)}-(\rho+\lambda) K\right) d s .
\end{aligned}
$$

Calculating the integral and simplifying results in

$$
A=K \frac{\rho+\lambda}{\rho+\lambda-\mu}=X_{c} .
$$

Replacing $b(t)$ in Equation (2) with $X_{c}$ now yields (the straightforward calculations are not presented)

$$
V(t, x)= \begin{cases}V_{E}(t, x) & \text { if } 0 \leq x<X_{c} e^{-\mu(T-t)} \\ V_{\infty}(x) & \text { if } x \geq X_{c} e^{-\mu(T-t)}\end{cases}
$$


This is our candidate solution. Note that $V \notin C^{1,1}$, so standard versions of Ito's formula for processes of finite variation will not work. In the proof below we do not need to use any version of Ito's formula in order to verify that $V$ is given by Equation (3), so this is not a problem.

Theorem 3.1 Let $X$ satisfy the SDE

$$
d X_{t}=\mu X_{t} d t-X_{t-} d N_{t}
$$

where $\mu>0$ and $N$ is a Poisson process with constant intensity $\lambda>0$. Then for $(t, x) \in[0, T] \times \mathbb{R}_{+}$

$$
V(t, x)=\sup _{\tau \in \mathcal{S}_{t, T}} E_{t, x}\left[e^{-\rho(\tau-t)} \max \left(X_{\tau}-K, 0\right)\right],
$$

where $\rho>0$ and $\rho+\lambda>\mu$, is given by

$$
V(t, x)= \begin{cases}0 & \text { if } 0 \leq x<K e^{-\mu(T-t)} \\ e^{-(\rho+\lambda)(T-t)}\left(x e^{\mu(T-t)}-K\right) & \text { if } K e^{-\mu(T-t)} \leq x<X_{c} e^{-\mu(T-t)} \\ \left(\frac{x}{X_{c}}\right)^{\frac{\rho+\lambda}{\mu}}\left(X_{c}-K\right) & \text { if } X_{c} e^{-\mu(T-t)} \leq x<X_{c} \\ x-K & \text { if } x \geq X_{c},\end{cases}
$$

where

$$
X_{c}=K \frac{\rho+\lambda}{\rho+\lambda-\mu} .
$$

An optimal stopping time at time $t \in[0, T]$ is given by

$$
\tau_{t}^{\star}=\inf \left\{s \in[t, T) \mid X_{s} \geq X_{c}\right\} \wedge T \text {. }
$$

Proof. Let $\hat{V}$ denote the function in $(\dagger)$, and let $V$ denote the optimal value. We will show that $\hat{V}=V$ and that $\tau_{t}^{\star}$ as given above is an optimal stopping time. Recall that, with $V_{\infty}$ and $V_{E}$ defined as above, for any $(t, x) \in[0, T] \times \mathbb{R}_{+}$

$$
\hat{V}(t, x)= \begin{cases}V_{E}(t, x) & \text { if } 0 \leq x<X_{c} e^{-\mu(T-t)} \\ V_{\infty}(x) & \text { if } x \geq X_{c} e^{-\mu(T-t)}\end{cases}
$$

Now define on $[0, T]$ the processes

$$
\begin{aligned}
U_{t} & =e^{-\rho t} \hat{V}\left(t, X_{t}\right), \\
M_{t} & =e^{-\rho t} V_{E}\left(t, X_{t}\right), \text { and } \\
Y_{t} & =e^{-\rho t} V_{\infty}\left(X_{t}\right) .
\end{aligned}
$$

Then

$$
U_{t}=M_{t} I_{t}+Y_{t}\left(1-I_{t}\right)
$$

where

$$
I_{t}=\mathbf{1}\left(0 \leq X_{t}<X_{c} e^{-\mu(T-t)}\right)
$$


Here $\left(M_{t}\right)$ is a martingale and $\left(Y_{t}\right)$ is a supermartingale with $Y_{t} \geq M_{t} P$-a.s. Furthermore $\left(I_{t}\right)$ is an increasing process, so for $0 \leq s \leq t$

$$
0 \leq\left(Y_{t}-M_{t}\right)\left(1-I_{t}\right) \leq\left(Y_{t}-M_{t}\right)\left(1-I_{s}\right) .
$$

We now get

$$
\begin{aligned}
E\left[U_{t} \mid \mathcal{F}_{s}\right] & =E\left[M_{t} I_{t}+Y_{t}\left(1-I_{t}\right) \mid \mathcal{F}_{s}\right] \\
& =E\left[M_{t}+\left(Y_{t}-M_{t}\right)\left(1-I_{t}\right) \mid \mathcal{F}_{s}\right] \\
& \leq M_{s}+\left(1-I_{s}\right) E\left[\left(Y_{t}-M_{t}\right) \mid \mathcal{F}_{s}\right] \\
& \leq M_{s}+\left(Y_{s}-M_{s}\right)\left(1-I_{s}\right) \\
& =U_{s} .
\end{aligned}
$$

Hence, $\left(U_{t}\right)$ is a supermartingale, and this implies

$$
E_{t, x}\left[e^{-\rho(s-t)} \hat{V}\left(s, X_{s}\right)\right] \leq \hat{V}(t, x)
$$

for any $0 \leq t \leq s \leq T$ and $x \in \mathbb{R}_{+}$. Since $\left(X_{t}\right)$ is right-continuous and $\hat{V}$ is a continuous function, the stochastic process $\left(e^{-\rho t} \hat{V}\left(t, X_{t}\right)\right)$ is a rightcontinuous supermartingale on $[0, T]$. Using the optional sampling theorem, see e.g. Theorem 17 in Chapter 2 of Protter [5], with $\tau \in \mathcal{S}_{t, T}$ yields

$$
E_{t, x}\left[e^{-\rho(\tau-t)} \hat{V}\left(\tau, X_{\tau}\right)\right] \leq \hat{V}(t, x) \text { for any } \tau \in \mathcal{S}_{t, T} .
$$

Since $\hat{V}(t, x) \geq \max (x-K, 0)$ we get

$$
E_{t, x}\left[e^{-\rho(\tau-t)} \max \left(X_{\tau}-K, 0\right)\right] \leq \hat{V}(t, x) \text { for any } \tau \in \mathcal{S}_{t, T},
$$

from which we conclude

$$
V(t, x)=\sup _{\tau \in \mathcal{S}_{t, T}} E_{t, x}\left[e^{-\rho(\tau-t)} \max \left(X_{\tau}-K, 0\right)\right] \leq \hat{V}(t, x)
$$

for any $(t, x) \in[0, T] \times \mathbb{R}_{+}$. To show that the reverse inequality also holds, we start by noting that

$$
V(t, x) \geq E_{t, x}\left[e^{-\rho\left(\tau_{t}^{\star}-t\right)} \max \left(X_{\tau_{t}^{\star}}-K, 0\right)\right]
$$

for every $(t, x) \in[0, T] \times \mathbb{R}_{+}$. We will now show that

$$
E_{t, x}\left[e^{-\rho\left(\tau_{t}^{\star}-t\right)} \max \left(X_{\tau_{t}^{\star}}-K, 0\right)\right]=\hat{V}(t, x) .
$$

(i) If $x \in\left[0, X_{c} e^{-\mu(T-t)}\right)$, then it is not possible to reach $X_{c}$. Hence, we will always get to $T$ before it is possible for $X$ to hit the level $X_{c}$, and we get $\tau_{t}^{\star}=T$ in this case. It follows that

$$
E_{t, x}\left[e^{-\rho\left(\tau_{t}^{\star}-t\right)} \max \left(X_{\tau_{t}^{\star}}-K, 0\right)\right]=V_{E}(t, x) \text { when } x \in\left[0, X_{c} e^{-\mu(T-t)}\right) .
$$


(ii) If $x \in\left[X_{c} e^{-\mu(T-t)}, X_{c}\right)$, then $X$ will reach the level $X_{c}$ if and only if the Poisson process does not jump on $\left[t, \tau_{t}^{\star}\right]$. If there is a jump of $X$ to 0 , then $\tau_{t}^{\star}=T$. Thus

$E_{t, x}\left[e^{-\rho\left(\tau_{t}^{\star}-t\right)} \max \left(X_{\tau_{t}^{\star}}-K, 0\right)\right]=E_{t, x}\left[e^{-\rho\left(\tau_{t}^{\star}-t\right)} \max \left(X_{\tau_{t}^{\star}}-K, 0\right) \mathbf{1}\left(\tau_{t}^{\star}<T\right)\right]$

when $x \in\left[X_{c} e^{-\mu(T-t)}, X_{c}\right)$. On $\left\{\tau_{t}^{\star}<T\right\}$ we have

$$
X_{\tau_{t}^{\star}}=X_{c}=x e^{-\mu\left(\tau_{t}^{\star}-t\right)} .
$$

It follows that

$$
\max \left(X_{\tau_{t}^{\star}}-K, 0\right)=X_{c}-K \text { and } e^{-\rho\left(\tau_{t}^{\star}-t\right)}=\left(\frac{x}{X_{c}}\right)^{\rho / \mu} \text { on }\left\{\tau_{t}^{\star}<T\right\}
$$

when $x \in\left[X_{c} e^{-\mu(T-t)}, X_{c}\right)$. Furthermore

$$
E_{t, x}\left[\mathbf{1}\left(\tau_{t}^{\star}<T\right)\right]=E_{t, x}\left[e^{-\lambda\left(\tau_{t}^{\star}-t\right)}\right]=\left(\frac{x}{X_{c}}\right)^{\lambda / \mu}
$$

in this case. Hence,

$$
E_{t, x}\left[e^{-\rho\left(\tau_{t}^{\star}-t\right)} \max \left(X_{\tau_{t}^{\star}}-K, 0\right)\right]=V_{\infty}(x)
$$

when $x \in\left[X_{c} e^{-\mu(T-t)}, X_{c}\right)$.

(iii) If $x \in\left[X_{c}, \infty\right)$, then $\tau_{t}^{\star}=t$ and

$$
E_{t, x}\left[e^{-\rho\left(\tau_{t}^{\star}-t\right)} \max \left(X_{\tau_{t}^{\star}}-K, 0\right)\right]=x-K
$$

when $x \in\left[X_{c}, \infty\right)$.

Together with the inequality in (4) this shows that

$$
V(t, x)=\hat{V}(t, x) \text { for every }(t, x) \in[0, T] \times \mathbb{R}_{+} .
$$

It also follows that $\tau_{t}^{\star}$ is an optimal stopping time at $t$.

Example 3.2 Consider the model with the following parameter values:

$$
\lambda=0.1, \rho=0.3, \mu=0.1, T=3 \text { and } K=2 .
$$

The value $V(0.5, x)$ as a function of $x \in \mathbb{R}_{+}$is shown in Figure 1. For $x \in$ $[0,2.077)$ this value is equal to $V_{E}(0.5, x)$ with $T=3$ and $K=2$, and for $x \in[2.077, \infty)$ the value is eqal to $V_{\infty}(x)$ with $K=2$. The level at which it is optimal to stop is $X_{c}=2.6675$. 


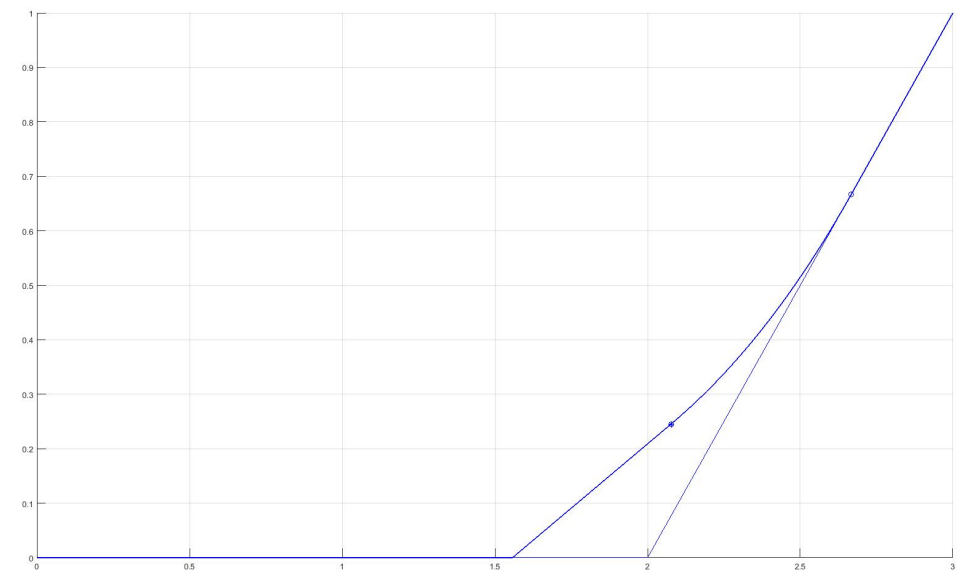

Figure 1: The option value when the parameter values are $\lambda=0.1, \rho=0.3$, $\mu=0.1, T=3, K=2$ and $t=0.5$. The filled circle to the left marks where the European option price and the perpetual option price meet (in this example at $\left.X_{c} e^{-\mu(T-t)}=2.077\right)$, and the circle to the right marks the level at which it is optimal to exercise the option ( $X_{c}=2.667$ in this example).

\section{Applications}

\subsection{Introduction}

In this section we keep the same modelling assumptions as in the main part of the paper, but we also add the assumption that the filtration is generated by the Poisson process, i.e. that

$$
\mathcal{F}_{t}=\sigma\left(N_{s}, 0 \leq s \leq t\right), t \in[0, T] .
$$

We assume that there exists a bank account with dynamics

$$
d B_{t}=r B_{t} d t \quad \text { with } B_{0}=1,
$$

and with constant interest rate $r>0$. Absence of arbitrage implies that there exists a probability measure $Q$ equivalent to $P$ and such that the discounted price of every price of a non-dividend paying traded asset when the risk-free rate is used as discount rate is a $Q$-martingale (see Jeanblanc et al [3] for details).

In our model, since the only traded asset is the bank account, every measure $Q$ equivalent to $P$ is a potential martingale measure. Since we assume that the filtration is generated by the Poisson process, the only way of changing the measure form $P$ to $Q$ is to change the intensity of the Poisson process (see Brémaud [1]). We assume that the intensity under $Q$ is a strictly positive 
constant $\lambda^{Q}>0$; hence $N$ is a Poisson process also under $Q$. The value of an American call option with time to maturity $T>0$ and strike price $K>0$ when the price of the underlying asset at time $t \in[0, T]$ is $X_{t}$, is given by

$$
V(t, x)=\sup _{\tau \in \mathcal{S}_{t, T}} E_{t, x}^{Q}\left[e^{-r(\tau-t)} \max \left(X_{\tau}-K, 0\right)\right] .
$$

(see Jeanblanc et al [3] and references therein for the theory). The dynamics of $X$ can be written

$$
d X_{t}=\mu X_{t} d t-X_{t-} d N_{t}=\left(\mu-\lambda^{Q}\right) X_{t} d t-X_{t-} d M_{t}^{Q},
$$

where

$$
M_{t}^{Q}=N_{t}-\lambda^{Q} t
$$

is a $Q$-martingale. We define the implied yield by

$$
\delta_{\mathrm{imp}}=r-\mu+\lambda^{Q},
$$

and using this the dynamics can be written

$$
d X_{t}=\left(r-\delta_{\mathrm{imp}}\right) X_{t} d t-X_{t-} d M_{t}^{Q} .
$$

In order to get a non-trivial solution, i.e. a solution in which the optimal stopping time is not equal to $T$ with probability 1 , we need to assume that

$$
r+\lambda^{Q}>\mu \Leftrightarrow \delta_{\mathrm{imp}}>0
$$

Using Theorem 3.1 we see that if the condition $\delta_{\text {imp }}>0$ is fulfilled, then the value at time $t \in[0, T]$ of the American call option is

$$
V(t, x)= \begin{cases}0 & \text { if } 0 \leq x<K e^{-\mu(T-t)} \\ e^{-\delta_{\mathrm{imp}}(T-t)}\left(x-K e^{-\mu(T-t)}\right) & \text { if } K e^{-\mu(T-t)} \leq x<X_{c} e^{-\mu(T-t)} \\ \left(\frac{x}{X_{c}}\right)^{1+\delta_{\mathrm{imp}} / \mu}\left(X_{c}-K\right) & \text { if } X_{c} e^{-\mu(T-t)} \leq x<X_{c} \\ x-K & \text { if } x \geq X_{c}\end{cases}
$$

where

$$
X_{c}=K\left(1+\mu / \delta_{\text {imp }}\right)
$$

If the asset modelled by $X$ pays out cash flows, e.g. dividend, then the dynamics under $Q$ will be different from the one given above. In the case of the asset delivering a constant yield $\alpha$, the implied yield will be

$$
\delta_{\text {imp }}=r-\mu+\lambda^{Q}+\alpha
$$

(see Dixit \& Pindyck [2]). Finally we remark that we can use a more general initial $P$-intensity $\lambda(t, \omega)$. As long as this $\lambda(t, \omega)$ is regular enough from a technical perspective, and we end up with a constant intensity $\lambda^{Q}$ under $Q$, the same result as above will hold. 


\subsection{A simple model of competition}

Consider a model where the value $V$ of a project after it has been initiated has dynamics

$$
d V_{t}=\mu V_{t} d t-V_{t-} d N_{t}
$$

under $P$. We have the possibility to invest in this asset by paying $K$ some time during $[0, T]$. This is an example of a real option; See Dixit \& Pindyck [2] for an extensive study. The fact that the value $V$ can drop to zero and stay there forever has the economical meaning that if we wait too long to invest, then a competitor can seize the opportunity, and the investment is no longer available for us. Hence, we can see this as a simple way of modelling competition. It has been previously been used in Reiss [6]; see also Vollert [7]. Given that the parameters are such that $\delta_{\mathrm{imp}}>0$, we can use the result in Section 4.1 above to get the value of this investment opportunity.

\subsection{Options written on a start-up company}

The value of a start-up company can go to zero if the sales of the product the company is selling is not in line with expectations. Here we consider the valuation of an American option with a finite time to maturity written on a non-listed start-up company. One way of modelling the stock price of such a company is to start with a geometric Browninan motion, and to this add the possibility of the stock price falling to zero and staying there. This could be achived by using the model

$$
d S_{t}=\mu S_{t} d t+\sigma S_{t} d W_{t}-S_{t-} d N_{t}
$$

for the stock price $S$. Here $\mu \in \mathbb{R}$ and $\sigma>0$ are two constants, $W$ is a onedimensional Brownian motion and $N$ is a Poisson process with constant intensity $\lambda>0$. As an approximation to this model we can set $\sigma=0$. This reflects the fact that the major risk for a start-up company is the risk of bankruptcy, not the daily fluctuations represented by the Wiener process, and leads to the model

$$
d S_{t}=\mu S_{t} d t-S_{t-} d N_{t} .
$$

We are now in the set-up described previously in this paper, and can again use the results in Section 4.1 to value the option.

If $S$ is the price of a traded asset, then we must assume that the asset pays out dividends in order to get a non-trivial solution. Since it is rare both that a start-up company pays dividends and that it is listed on a stock exchange, the incomplete market setting is probably the most realistic one.

\section{References}

[1] Brémaud, P. (1981), 'Point Process and Queues: Martingale Dynamics', Springer-Verlag. 
[2] Dixit, A. K. \& Pindyck, R. S. (1994), 'Investment under Uncertainty', Princeton University Press.

[3] Jeanblanc, ;., Yor, M. \& Chesney M. (2009), 'Mathematical Methods for Financial Markets', Springer-Verlag.

[4] Peskir, G. \& Shiryaev, A. (2006), 'Optimal Stopping and Free-Boundary Problems', Birkhäuser.

[5] Protter, P. E. (2004), 'Stochastic Integration and Differential Equations', Springer-Verlag.

[6] Reiss, A. (1998), 'Investment in Innovations and Competition: An Option Pricing Approach', The Quarterly Review of Economics and Finance, Vol. 38 , Special Issue, p. 635-650.

[7] Vollert, A. (2003), 'A Stochastic Control Framework for Real Options in Strategic Evaluation', Birkhäuser. 